\title{
Gestión actual del talento humano: contexto universitario
}

\section{Current management of human talent: university context}

Doi: $10.22458 /$ rna.v12i2.3914

Mag. Ana Lorena Carvajal-Pérez

Universidad Estatal a Distancia, Unidad Servicios al Personal, San José, Costa Rica;

lcarvajal@uned.ac.cr,

https://orcid.org/0000-0003-2321-5496

\section{RESUMEN}

Este artículo académico es el resultado del análisis y evaluación de lo que representa la gestión del talento humano en el entorno actual. Su objetivo es plantear la nueva visión de la gestión, a partir de una revisión bibliográfica sobre la administración de recursos humanos y su evolución. Se evalúa la importancia de su transformación, desde la perspectiva de los avances organizacionales, y se concluye que se requiere un cambio paradigmático en la gestión del talento humano, con miras a incrementar el aporte estratégico que puede brindar en la consecución de objetivos y metas organizacionales.

\section{ABSTRACT}

This work is the result of the analysis and evaluation of what human talent management represents in the current work environment. The main goal is to set a new way of viewing management, starting from a bibliographic review of human resources management, and its evolution through time, highlighting the importance of its transformation, going through organizational progress, and concluding that a change in human talent management its required, in order to increase the strategical support, it can bring to organizational goals achievement.

\section{RÉSUMÉ :}

Cet article est le résultat de l'analyse et de l'évaluation de ce que représente la gestion des talents humains dans l'environnement actuel. Son objectif est de formuler la nouvelle vision de la gestion, à partir d'une revue bibliographique sur l'administration des ressources humaines et son évolution. Il évalue l'importance de sa transformation du point de vue des progrès organisationnels et conclut qu'un changement de paradigme dans la gestion des talents humains est nécessaire, en vue d'accroître sa contribution stratégique à la réalisation des buts et objectifs de l'organisation.

\section{RESUMO:}

Este artigo acadêmico é o resultado da análise e avaliação do que a gestão do talento humano representa no ambiente atual. Seu objetivo é plantear a nova visão da gestão, a partir de uma revisão bibliográfica sobre a administração de recursos humanos e sua evolução. Avalia-se a importância da sua transformação, da perspectiva dos avanços organizacionais, e conclui-se que é necessária uma mudança paradigmática na gestão do talento humano, com vista a aumentar a contribuição estratégica que pode proporcionar na consecução dos objetivos e objetivos organizacionais.

\section{PALABRAS CLAVE:}

GESTIÓN DE TALENTO HUMANO, ADMINISTRACIÓN DE RECURSOS HUMANOS, TRANSFORMACIÓN DE LA GESTION, ADMINISTRACIÓN DE TALENTOS, ESTRUCTURA ORGANIZACIONAL, TENDENCIAS DE LA FUERZA LABORAL

\section{KEY WORDS:}

HUMAN TALENT MANAGEMENT, HUMAN RESOURCES MANAGEMENT, TRANSFORMATION MANAGEMENT, TALENT MANAGEMENT, ORGANIZATIONAL STRUCTURE, WORKFORCE TENDENCIES.
MOTS-CLÉS :

GESTION DES TALENTS HUMAINS, GESTION DES RESSOURCES HUMAINES, TRANSFORMATION DE LA GESTION, GESTION DES TALENTS, STRUCTURE ORGANISATIONNELLE, TENDANCES DE LA MAIND'OEUVRE

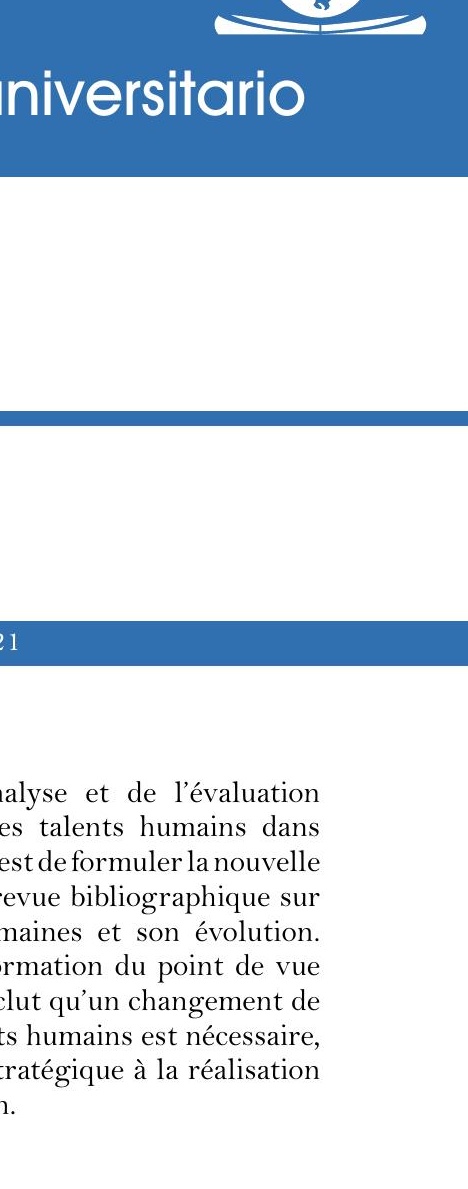




\section{INTRODUCCIÓN}

El siglo XXI plantea grandes retos para las organizaciones. Aspectos como la escasez de recursos, la globalización, la diversidad, los avances tecnológicos, la fuerte competencia, la exigencia cada vez mayor por la implementación de estándares de calidad y excelencia en la producción de bienes y servicios, el acceso a la información, la difusión masiva, entre otros, obligan a replantear los procesos laborales, con miras a incorporar acciones que permitan dar una respuesta ágil y oportuna a lo que la sociedad espera de la gestión.

Las personas, otrora vistas como apéndice de los procesos laborales detrás de maquinaria o equipos, en la actualidad ocupan un rol esencial en la consecución de los objetivos y metas, y por ende son corresponsables del éxito organizacional. Es por ello que los procesos inherentes a la administración del talento han tenido que transformarse, con el fin de poder adaptarse a las nuevas condiciones que el entorno exige. La vieja estructura de departamentos de recursos humanos, orientados principalmente al trámite de contrataciones, pago de nómina y seguridad laboral, ha tenido que abrir paso a una nueva forma de gestionar el talento, ocupando una posición más estratégica, como acompañamiento y asesoría para promover una eficaz toma de decisiones organizacional.

Chiavenato (2011) plantea que todos estos cambios del entorno generan grandes retos para el logro de una gestión efectiva de las personas. No obstante, presenta como el primer gran desafío lo siguiente:

Antes el área de RH se caracterizaba por definir políticas en la que las personas recibían un trato genérico y estandarizado. Las técnicas de RH consideraban homogéneas a todas las personas. Hoy en día, cada vez se toman más en cuenta estas particularidades: la administración de Recursos Humanos hace hincapié en las diferencias individuales y en la diversidad en las organizaciones. La razón es sencilla cuanto mayor es la diferencia entre las personas, tanto mayor su potencial e innovación. (p. 39)

En este nuevo rol que las personas ocupan dentro de las organizaciones, la comprensión de los factores que orientan su accionar hacia la consecución de los resultados esperados adquiere mucha importancia. Bajo esta premisa, para lograr el éxito organizacional, como condición sine qua non, se debe asumir que las personas son el centro de la gestión y tener conciencia de la importancia de sus capacidades. Chiavenato (2020) define el talento como las capacidades, habilidades y otras características que deben ser validadas y compensadas continuamente. No obstante, advierte sobre la importancia de su abordaje integral al indicar que: "no se puede abordar el talento de forma aislada como un sistema cerrado. Solo no llega lejos, pues debe existir y coexistir en un contex to que le permita libertad, autonomía y cobertura para poder expandirse" (p. 41).

Es por ello que, adicionalmente a esta visión de persona como motor de éxito, existen otros factores que intervienen en el ámbito organizacional que de igual forma deben ser evaluados y alineados, de modo que se pueda asegurar el cumplimiento de los objetivos propuestos.

Este artículo tiene como objetivo plantear los cambios esenciales que debe realizar la gestión universitaria de talento humano, con el fin de adaptar su accionar a los cambios que el entorno actual plantea, y colaborar como socio estratégico para la consecución del éxito en los procesos académicos.

\section{DE ADMINISTRAR PERSONAL A GESTIONAR TALENTOS}

La necesidad de administrar los procesos inherentes a las personas dentro de las organizaciones nace en la primera mitad del siglo XX, producto del creciente auge de la industrialización. Se tenía la idea de que el éxito organizacional estaba centrado en las máquinas, además se consideraba a las personas como parte del proceso de producción y, por ende, era necesario establecer políticas orientadas a resguardar el lugar de trabajo, con el fin de evitar accidentes laborales. Ariza-Montes et al. (2004) establecen esta época como "etapa administrativa” de la gestión de talento humano, por cuanto su función general estaba orientada hacia la elaboración de contratos de trabajo, control de jornadas y aseguramientos de riesgos.

En la segunda mitad del siglo XX se inicia una etapa de cambios vertiginosos en el mundo organizacional. La incorporación de la tecnología en los procesos laborales modificó por completo no solo la forma de generar resultados, sino que transformó la manera en que se realizan las tareas en cada uno de los puestos de trabajo. Dolan et al. (2010) indican que uno de los efectos directos del ingreso de la tecnología a la actividad laboral fue la incorporación de estructuras cada vez menos jerarquizadas, dada la necesidad de generar un agrupamiento de la actividad por procesos. 
Otro de los cambios más grandes que se dieron en esta época fue la incorporación de la mujer a la educación y al trabajo. Werther et al. (2019) señalan que esto se produjo debido a dos factores esenciales: "la extensión de la educación general y el incremento de la demanda de personas que necesitaban integrarse a la economía moderna" (p. 32). Se crean en este período los departamentos de recursos humanos, para generar procesos de diseño de puestos, reclutamiento, selección, capacitación y remuneración, lo cual produce una mayor especialización en el área. Si bien las personas son vistas como parte importante del engranaje empresarial, la inversión estaba centrada en la tecnología como factor esencial del éxito organizacional.

El siglo XXI plantea el gran reto para la administración de talento humano: la gestión del conocimiento. FernándezLópez (2005) define el conocimiento como: "El conjunto de información desarrollado en el contexto de una experiencia y transformado a su vez en otra experiencia” (p. 205). El desafío que esto representa está relacionado con "redefinir el concepto de aprendizaje corporativo para convertirlo en un proceso que comprende desde el desarrollo hasta la aplicación y explotación del conocimiento necesario en la cobertura de un objetivo concreto” (p. 207).

Dentro de este contex to, las funciones esenciales del talento humano deberán estar dirigidas a promover el desarrollo profesional y personal, generar espacios de aprendizaje y definir puestos de trabajo orientados hacia la creatividad e innovación, que permitan a las personas desplegar todo su potencial para obtener los resultados que se esperan. Chiavenato (2020) indica que:

Es el turno del conocimiento, de las habilidades y de las competencias esenciales del capital humano y del capital intelectual. El conocimiento se ha convertido en el factor básico y el desafío mayor será estimular la productividad del conocimiento para generar las competencias. Lograr que el conocimiento sea útil y productivo, pasó a ser la mayor responsabilidad de los gerentes. Y el trabajo manual fue sustituido por el trabajo mental y las máquinas inteligentes. El conocimiento se tornó completo y virtual, y hoy es la moneda más valiosa del planeta. Puede estar en documentos, registros, patentes, proyectos, bancos de datos, pero antes debe estar en el cerebro de las personas en forma de habilidades, actitudes y competencias para después aplicarlas. Estamos hablando de talento humano. (p. 31)

Sin embargo, esta visión de lo que representa la administración de recursos humanos se da hasta que se define a la persona como fuente de éxito organizacional. Ariza-Montes et al. (2004) sintetizan en la tabla 1 la transición que ha sufrido la gestión de talento humano: 
Tabla 1. Evolución de la función de los recursos humanos en el siglo XX

\begin{tabular}{|c|c|c|c|c|c|}
\hline \multicolumn{3}{|c|}{ Etapa administrativa } & $\begin{array}{c}\text { Etapa } \\
\text { psicotécnica }\end{array}$ & Etapa laboral & Etapa de RRHH \\
\hline \multirow{11}{*}{ 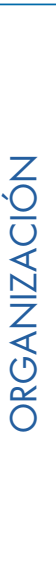 } & Finalidad & CANTIDAD & EFICIENCIA & \multirow{3}{*}{$\begin{array}{l}\text { REDUCCIÓN DE COSTES } \\
\text { Optimización de resultados }\end{array}$} & CALIDAD \\
\hline & & Mantenimiento & & & Mejora \\
\hline & & de la producción & & & continua \\
\hline & & & & \multirow{2}{*}{$\begin{array}{l}\text { TÁCTICA } \\
\text { Director funcional }\end{array}$} & de procesos \\
\hline & Posición y & OPERATIVA & TÁCTICA & & ESTRATÉGICA \\
\hline & relevancia & Dependencia & Directivo & \multirow{6}{*}{ Derecho del trabajo } & Staff del director \\
\hline & administrativa & del director & de segundo & & general o directivo \\
\hline & & administrativo & nivel & & de primer nivel \\
\hline & Conocimientos & Económico- & Psicología & & Planificación \\
\hline & relevantes & financieros & & & estratégica \\
\hline & & y legales & & & \\
\hline \multirow{3}{*}{$\begin{array}{l}\text { O } \\
Z \\
\text { o } \\
\bigcirc \\
\frac{V}{Z}\end{array}$} & $\begin{array}{l}\text { Contexto } \\
\text { económico }\end{array}$ & Estabilidad & Expansión & Crisis & Competitividad elevada \\
\hline & $\begin{array}{l}\text { Cualificación } \\
\text { del personal } \\
\text { operativo }\end{array}$ & $\begin{array}{l}\text { Escasa (dirección de } \\
\text { operarios) }\end{array}$ & $\begin{array}{l}\text { Media moderada } \\
\text { (dirección de } \\
\text { empleados) }\end{array}$ & $\begin{array}{l}\text { Profesionalización media } \\
\text { moderada (dirección de } \\
\text { empleados) }\end{array}$ & $\begin{array}{l}\text { Elevada } \\
\text { profesionalización }\end{array}$ \\
\hline & $\begin{array}{l}\text { Valores culturales } \\
\text { básicos }\end{array}$ & $\begin{array}{l}\text { Producción } \\
\text { cuantitativa, fidelidad, } \\
\text { conformidad } \\
\text { cumplimiento, } \\
\text { disciplina }\end{array}$ & $\begin{array}{l}\text { Racionalización } \\
\text { motivación, eficiencia, } \\
\text { medición de resultados }\end{array}$ & Movilidad, flexibilidad & $\begin{array}{l}\text { Desarrollo, } \\
\text { participación, } \\
\text { aprendizaje continuo, } \\
\text { creatividad }\end{array}$ \\
\hline
\end{tabular}

Fuente: Ariza-Montes et al. (2004, p. 7).

Al observar en esta figura la evolución que ha tenido la gestión de talento humano, es fácil comprender la importancia que reviste la implementación de políticas orientadas al desarrollo y aprendizaje de las personas, como factor clave para el logro de los objetivos, de las metas y, en resumen, del éxito organizacional.

\section{LA GESTIÓN DE TALENTO HUMANO EN EL CONTEXTO ACTUAL}

Son muchos los retos que se deben enfrentar a la hora de gestionar el talento humano en la actualidad. Dessler y Varela (2017) brindan importantes aportes con respecto a los grandes desafíos a los que se enfrentan las organizaciones para gestionar de manera efectiva su talento, entre ellos:

a) Los avances tecnológicos. Estos no solo generan un cambio en el análisis y diseño de puestos, sino en la forma en que se realiza la captación del talento. Por un lado, los trabajos operativos pueden ser realizados de forma más ágil y exacta con la utilización de la tecnología. Pero, a su vez, esto podría implicar la creación de otras ocupaciones o transformar los puestos actuales en términos de funciones, responsabilidad o requerimientos en cuanto al perfil profesiográfico que se ajusta a las nuevas tendencias.

En este sentido, Werther y Davis (2005), advierten que:

La inteligencia artificial, el manejo de datos y los ambientes virtuales ayudarán a la mayoría de las compañías e instituciones a resolver problemas con mayor rapidez y con mayor calidad. Es evidente que, si la empresa tiene acceso a nuevas tecnologías, el departamento de recursos humanos debe estudiar los posibles efectos que su introducción tendrá sobre su capital humano. (p. 46) 
En el documento de MIDEPLAN (2020) se refuerza el cambio que se debe realizar en los perfiles de los puestos, producto del cambio en la tecnología, al mencionar:

Las habilidades transversales o blandas ahora están tomando una necesidad muy fuerte en el sector empleador, las cuales debe ser desarrolladas por parte de las personas en los niveles básicos de la educación. Algunas de estas habilidades son: trabajar con datos y tomar decisiones basadas en datos; resolver problemas complejos; persuadir, inteligencia emocional y enseñar a otros: cognitivas (creatividad, pensamiento matemático) y procedimentales (comunicación, escuchar ideas de otros y pensamiento crítico), así como otras no cognitivas como la capacidad de socialización o la resiliencia, trabajo equipo y liderazgo. (p. 22)

Es imprescindible reconocer el papel preponderante que tienen los departamentos de recursos humanos como facilitadores del cambio, ya que orientan su accionar a lograr que las empresas e instituciones actualicen sus procesos de trabajo y, de esta manera, puedan enfrentar con éxito las nuevas condiciones que el entorno plantea. Por ello, es mediante las personas que se logran los objetivos organizacionales, lo cual se convierte en la oportunidad para la gestión de talento humano de dar un valor estratégico al aporte que brinda a nivel general.

b) Tendencias de la naturaleza del trabajo. Al ritmo en que las organizaciones evolucionan, se deben desarrollar los puestos de trabajo. Si bien la dinámica natural empresarial debe ser hacia la transformación, con el fin de adaptase a los cambios del entorno, el avance, lamentablemente, a veces no ha ido a la misma velocidad. Las razones pueden ir desde la lentitud en la toma de decisiones, hasta impedimentos de tipo financiero. No obstante, a partir del año 2020 esta situación cambió producto de los efectos que tuvo la pandemia por COVID-19 en los procesos de trabajo. Durante este período las empresas se vieron obligadas a implementar el trabajo a distancia, mediado por la tecnología, conocido como teletrabajo.

Sin embargo, en la investigación realizada por Tapasco-Alzate y Girado-García (2020), concluyeron que las grandes resistencias a las que se enfrenta una implementación efectiva del teletrabajo son: 


\section{Tabla 2.Resistencias a la implementación del teletrabajo}

\begin{tabular}{|c|c|c|}
\hline Dificultades & Argumentos & Referencias \\
\hline $\begin{array}{l}\text { Pérdida de control sobre los } \\
\text { teletrabajadores }\end{array}$ & $\begin{array}{l}\text { La separación física ocasionada por la } \\
\text { realización del trabajo remoto genera dificultades } \\
\text { en la coordinación y pérdida de control de los } \\
\text { empleados. }\end{array}$ & $\begin{array}{l}\text { Bailey y Kurland (2002); Peters y Dulk } \\
\text { (2003); Pérez et al. (2005); Mello (2007); } \\
\text { Pérez y Gálvez (2009); Tureken et al. } \\
\text { (2011); Dutcher (2012). }\end{array}$ \\
\hline $\begin{array}{l}\text { Medición del desempeño de } \\
\text { los trabajadores }\end{array}$ & $\begin{array}{l}\text { Dado que los métodos de valoración del } \\
\text { desempeño se basan en la supervisión presencial, } \\
\text { se hace necesario buscar formas alternativas de } \\
\text { evaluación que apunten al cumplimiento de los } \\
\text { objetivos. }\end{array}$ & $\begin{array}{l}\text { Topi (2004); Dickson y Clear (2006); Mello } \\
\text { (2007); Pearce (2009; Burbach y Day } \\
\text { (2012). }\end{array}$ \\
\hline $\begin{array}{l}\text { Impacto sobre el equipo de } \\
\text { trabajo }\end{array}$ & $\begin{array}{l}\text { Al no estar el empleado físicamente presente, } \\
\text { se afecta la interacción con los colegas y } \\
\text { supervisores. }\end{array}$ & $\begin{array}{l}\text { Topi (2004); Pérez et al. (2005); Mello } \\
\text { (2007); Pearce (2009); Davis (2012). }\end{array}$ \\
\hline Seguridad de la información & $\begin{array}{l}\text { Los riesgos de extracción ilegal de información } \\
\text { confidencial se incrementan cuando los } \\
\text { empleados tienen acceso desde sus residencias a } \\
\text { dicha información. }\end{array}$ & $\begin{array}{l}\text { Peters et al. (2004); Pérez et al. (2005); } \\
\text { Dickson y Clear (2006); Mello (2007); Kim } \\
\text { (2018). }\end{array}$ \\
\hline $\begin{array}{l}\text { Costos de implementación y } \\
\text { acceso a la tecnología }\end{array}$ & $\begin{array}{l}\text { Para el buen desarrollo de un programa de } \\
\text { implementación es indispensable disponer de las } \\
\text { herramientas tecnológicas apropiadas. }\end{array}$ & $\begin{array}{l}\text { Pérez et al. (2005); Pearce (2009); } \\
\text { Valmohammadi (2012). }\end{array}$ \\
\hline Selección de empleados aptos & $\begin{array}{l}\text { Un buen trabajador no es necesariamente un } \\
\text { buen teletrabajador, es primordial que posea } \\
\text { ciertas habilidades y conocimientos, así como } \\
\text { buena disposición hacia el teletrabajo. }\end{array}$ & $\begin{array}{l}\text { Peters et al. (2004); Pérez et al. (2005); } \\
\text { Mello (2007); Turetken et al. (201 1); Burbach } \\
\text { y Day (2012); Ahmed et al. (2014). }\end{array}$ \\
\hline Rechazo entre los empleadores & $\begin{array}{l}\text { Desconfianza hacia el comportamiento } \\
\text { de los empleados fuera de la supervisión } \\
\text { física. Preocupación por que los desafios de } \\
\text { coordinación incrementen su carga laboral. }\end{array}$ & $\begin{array}{l}\text { Pérez et al. (2005); Mello (2007); Turetken } \\
\text { et al. (2011); Neufeld et al. (2010); Bloom et } \\
\text { al. (2012); Kirs y Bagchi (2012). }\end{array}$ \\
\hline $\begin{array}{l}\text { Resistencia al cambio de las } \\
\text { estructuras organizacionales }\end{array}$ & $\begin{array}{l}\text { Implica un cambio de perspectiva en la } \\
\text { cultura del trabajo tradicional soportada en la } \\
\text { presencialidad. }\end{array}$ & $\begin{array}{l}\text { Illegems y Verbeke (2004); Pérez et al. } \\
\text { (2005). }\end{array}$ \\
\hline Temor a la incertidumbre & $\begin{array}{l}\text { El desconocimiento sobre el concepto de } \\
\text { teletrabajo o sus beneficios potenciales conlleva a } \\
\text { tener recelo en su adopción. }\end{array}$ & $\begin{array}{l}\text { Peters y Dulk (2003); Pérez et al. (2005); } \\
\text { Dickson y Clear (2006). }\end{array}$ \\
\hline Aspectos culturales & $\begin{array}{l}\text { Las dinámicas de adopción también se ven } \\
\text { matizadas por aspectos culturales. }\end{array}$ & $\begin{array}{l}\text { Peters y Dulk (2003); Topi (2004); } \\
\text { Valmohammadi (2012). }\end{array}$ \\
\hline
\end{tabular}

Fuente: Tapasco-Alzate y Giraldo-García (2020).

Si bien la pandemia determinó un teletrabajo forzado, son innegables los beneficios que esta modalidad laboral puede representar para las organizaciones y para las personas. Por ello, se convierte en un gran reto para la gestión de talento humano promover acciones orientadas a:

- Replantear los procesos laborales, con el fin de adaptar las actividades a las nuevas condiciones mediadas por las TIC. Asimismo, actualizarlas a las nuevas circunstancias y exigencias del entorno.

- Establecer las condiciones o competencias que deben cumplir las personas para asegurar el éxito en el trabajo a distancia.

- Promover a nivel organizacional canales de comunicación efectivos, por medio del uso de la virtualidad, con miras a fortalecer los vínculos en los equipos de trabajo.

- Determinar las actividades que pueden ser realizadas de manera remota, con el fin de ajustar las descripciones de los puestos.

Todo lo anterior se considera necesario para una implementación eficaz del teletrabajo a nivel organizacional.

c) Cambios en la fuerza laboral. Son variadas las razones por las cuales se ha diversificado la fuerza laboral a lo interno de las organizaciones. Bohlander y Snell (2008) mencionan la globalización que trajo consigo la migración laboral, lo cual enriqueció el ámbito laboral con la convergencia de culturas, intereses, 
preferencias, entre otros. Por su parte, Chiavenato (2020) menciona como una de las causas, "la sustitución de una economía sustentada en la manufactura y la explotación de recursos naturales por una economía basada en el valor del conocimiento, la información y la innovación” (p. 32), lo que ha llevado a una mayor conciencia sobre el talento y las competencias de las personas como los grandes valores organizacionales.

Con lo anterior, el gran reto del mundo laboral actual es cómo lograr la ventaja competitiva por medio de las personas. Namdarian et al. (2020) indican que la combinación del conocimiento con otros factores organizacionales (estructura, recursos y estrategia), resultan la clave para crear valor individual y colectivo, con el fin de obtener empresas e instituciones innovadoras, creativas y eficaces.

d) La estructura organizacional. La eficacia y la eficiencia empresarial tienen cimentado el éxito en la forma en que se definen los procesos, las actividades y tareas de la organización, y de cómo estas se orientan a la consecución de los resultados con estándares de calidad y excelencia, mediante mecanismos de comunicación e información fluidos entre todas las partes que intervienen en el ámbito laboral. MurilloVargas et al. (2019) indican lo siguiente:

La estructura organizativa evidencia el diseño formal de roles que permite integrar las funciones, flujos de trabajo y líneas de autoridad, en función de los objetivos establecidos por una organización. Las estructuras organizativas son orgánicas cuando existe descentralización en la toma de decisiones, comunicaciones abiertas y mayor flexibilidad. Por el contrario, las estructuras mecanicistas suelen tener mayor control y rigurosidad en los procesos normativos, el flujo de información y centralización en la toma de decisiones. (p. 22)

Durante mucho tiempo en las organizaciones se apostó por la implementación de estructuras tradicionales, rígidas, divididas esencialmente por la especialización de procesos y nivel de jerarquía en la cadena de mando. Hoy, el diseño de una estructura debe contemplar aspectos que se orienten al éxito. Robbins y Judge (2017) indican que estos elementos a considerar en el diseño de la estructura son:

- La estrategia. Tiene relación directa con la creación de valor. Define la metodología que se llevará a cabo y las propuestas organizacionales para cumplir con los objetivos.

- Tamaño de la organización. Debe analizarse el tamaño de la empresa para definir cuál modelo de diseño de estructura se ajuste más, con el fin de procurar los efectos de los procesos de trabajo.

- Tecnología. La manera en que se utiliza la tecnología para transformar los insumos en resultados, así como el grado de dependencia de esta para la eficacia de los procesos laborales, debe ser un punto a analizar en la definición de la estructura, con el fin de promover niveles de coordinación y supervisión orientados al éxito organizacional.

- Ambiente. Para el diseño de una estructura eficiente es necesario tomar en cuenta la estabilidad en que se desarrolla el sector en el cual se mueve la empresa, el crecimiento que soporta para una generación de resultados eficiente y la complejidad referida a la forma en que convergen los métodos para la consecución de objetivos, normativa interna y externa atinente al área de acción y el tipo de competencia a la que se enfrenta en el mercado.

- Instituciones: Robbins y Judge (2017) definen este criterio como "factores culturales que provocan que muchas organizaciones tengan estructuras similares, sobre todo los factores que quizá no conduzcan a consecuencias adaptativas" (p. 513).

\section{GESTIONAR EL TALENTO EN EL CONTEXTO UNIVERSITARIO ESTATAL}

El acelerado avance de la tecnología y su incorporación en los procesos laborales ha modificado no solo la forma en que se realiza el trabajo y sus resultados, sino que también ha producido un cambio en el acceso a la información y, por ende, transformó por completo las necesidades de la humanidad. Bindé (2005) menciona:

En las sociedades del conocimiento, los valores y prácticas de creatividad e innovación desempeñarán un papel muy importante, aunque sea por su capacidad de poner en tela de juicio a las nuevas necesidades de la sociedad. La creatividad y la innovación conducen asimismo a promover procesos de colaboración de nuevo tipo que hayan dado resultados especialmente fructíferos. (p. 19) 
Desde esta perspectiva, Zurbriggen y González-Lago (2014) indican:

El gran desafío de las sociedades actuales se encuentra en la capacidad de desarrollar nuevas formas de trabajar colaborativamente y nuevas formas de gestionar el conocimiento (diálogo de saberes), con el objetivo de construir una visión-acción común para resolver problemas públicos con una lógica transformadora. Ello demanda nuevos abordajes transdisciplinarios, multiactorales y multisectoriales y, por lo tanto, integrales, dejando de lado los enfoques dicotómicos (Estado versus mercado versus sociedad, competitividad versus equidad versus medio ambiente) por abordajes articulados entre los distintos actores intervinientes. Para esto se requiere un cambio de paradigma en cuanto al enfoque y al uso de nuevas metodologías que permitan comprender y entender la realidad y, por lo tanto, para construir el conocimiento. (p. 341)

En este contex to, las universidades toman un rol esencial como agentes de transformación y promotoras del desarrollo social. Rodríguez-Ponce (2009) describe con claridad el papel que tienen las universidades y su contribución a la movilidad social y equidad en la sociedad del conocimiento:

La sociedad de conocimiento y la era de la globalización imponen a los sistemas universitarios ciertos desafíos centrales para las instituciones ubicadas en cualquier parte del mundo. Primero, las instituciones universitarias deben constituirse en un elemento básico para generar mayores niveles de competitividad en el país. En efecto, en la sociedad del conocimiento y en un ambiente globalizado la formación de capital humano, avanzado, así como la investigación, el desarrollo y la innovación constituyen pilares fundamentales de la ventaja competitiva para la nación sus organizaciones. Segundo, las instituciones universitarias deben constituirse en una fuente esencial de oportunidades de formación continua y movilidad social. (p. 826)

Esta función esencial de las universidades como potenciadores del desarrollo social - lo cual representa su valor público- requiere de políticas internas que generen eficiencia y eficacia en su quehacer. Como se ha indicado a lo largo de este artículo, las personas constituyen el eje central para el logro de los objetivos de la organización, por lo que es de vital importancia analizar cuáles deben ser las prácticas comunes para gestionar el talento en una institución de educación superior, con miras a obtener, por medio del capital humano, los elementos distintivos que permitan lograr el éxito en cada una de las áreas que la componen.

\section{RESULTADO DEL ANÁLISIS Y APLICACIÓN EN EL CONTEXTO UNIVERSITARIO}

A continuación, se presentan los resultados de este análisis, distribuidos por área de interés dentro de la gestión de talento humano en el ámbito universitario:

\section{Cultura organizacional}

Para Robbins y Judge (2017) existen siete características por las que una organización se distingue de otras (elemento diferenciador competitivo), mediante las cuales se puede ver su esencia; estas son: "innovación y toma de riesgos, atención a los detalles, orientación a la gente, orientación a los equipos, dinamismo y estabilidad" (p. 527). Para cumplir su misión, las universidades deben promover ambientes laborales en los que las personas puedan desarrollarse, y se les permita la incorporación de ideas innovadoras y creativas, y en los que se les faculte para la toma de decisiones en su ámbito de acción.

Para la consecución de este fin, la gestión de talento humano debe preocuparse por promover liderazgos institucionales que susciten en sus equipos de trabajo las siguientes actitudes:

- Visión a futuro.

- Empoderamientos.

- Libertad para crear e innovar.

- Mantener una conducta congruente con los principios y valores organizacionales.

- Búsqueda de soluciones para cumplir metas.

- Aprendizaje.

Para generar lo anterior, las universidades deben procurar procesos estrictos de selección de sus líderes en las diferentes áreas, con el fin de contar con personas que se destaquen por: dominio teórico de su disciplina, ética, 
vocación de servicio, deseo continuo de aprendizaje, liderazgo participativo, empatía, coherencia y disciplina. Adicionalmente, desde la gestión de talento humano se pueden establecer programas continuos para promover estos saberes, principios y valores, para mantener actualizados sus liderazgos.

\section{Estructura organizacional}

La forma en que se vinculen todos los elementos que intervienen en la consecución de la estrategia organizacional se convierte en un factor clave para el logro de los resultados que se esperan. Del análisis de la estructura de una institución se puede comprender su esencia, su forma de gestionar las actividades, sus líneas de autoridad y la forma de organizarse para generar sus servicios. En una institución de educación superior, la estructura debe estar diseñada de manera que promueva: disposición de actividades (división del trabajo) destinadas al cumplimiento de la misión, canales efectivos de comunicación y coordinación entre los elementos que convergen, procesos académicos con excelencia, orientación a la calidad, compromiso con la investigación, procesos efectivos de planificación, una adecuada gestión financiera, políticas definidas de talento humano y aplicación de procesos de evaluación institucional orientados hacia la mejora continua.

En síntesis, es de vital relevancia en el contexto universitario la implementación de una estructura orientada a la consecución de sus objetivos con altos estándares de excelencia, que cumpla con las expectativas del país y que promueva el desarrollo social que es su fin último.

\section{Gestión de talento humano como facilitador de procesos esenciales universitarios}

Planeación del talento humano. El proceso de planeación tiene dos etapas. La primera, relacionada con las características que deben tener las personas para incorporarse de manera efectiva a una organización, es decir, todas aquellas particularidades que las distinguen y las orientan a cumplir con la estrategia. La segunda, la cantidad de personas que se requieren para el cumplimiento eficaz de su razón de ser. Para que ambas etapas sean exitosas es imprescindible como primer paso que exista una definición muy clara de su misión, su visión, sus objetivos estratégicos y que, de manera expresa, se identifiquen los principios y valores que fundamentan su cultura. Finalmente, es necesaria una efectiva estructuración de procesos de trabajo para la consecución del propósito de creación.

Una propuesta para definir los factores que se tienen que tomar en cuenta para planificar el talento humano es dividirlos en: 1) Factores externos: orientación social y productiva del país, tendencias del mercado laboral (internacional y nacional) que orientan las necesidades de formación o capacitación, políticas gubernamentales, contexto en que se encuentra la educación y la situación de la economía, tanto del país como de la organización. 2) Factores internos: oferta del mercado en los diferentes puestos, grado de mediación de la tecnología en los procesos de trabajo, nivel de especialización requerido, evaluación continua de cambios en la demanda de conocimientos, habilidades o destrezas, niveles y tipos de ausentismos e índices de rotación.

La gestión de talento humano debe acompañar de manera estratégica estos procesos de definición, con el fin no solo de proporcionar información de la situación país y del mercado laboral que sustente la toma de decisiones, sino para alinear de manera efectiva el resto de procesos atinentes al área, de forma que exista coherencia entre las políticas organizacionales y la gestión de talento humano. Además, como función inherente a su actividad, debe establecer canales de comunicación e información, con el fin lograr que las personas interioricen la cultura y las decisiones organizacionales.

Análisis ocupacional y diseño de puestos. En el ámbito universitario gestionar el talento humano debe partir del conocimiento como fuente esencial para generar ventaja estratégica, ya que es un factor central para la innovación y la creatividad organizacional, en el que se mezclan las necesidades de aprendizajes con las experiencias vividas en el ámbito institucional. Desde esta perspectiva, la gestión por competencias se convierte en un proceso primordial para implementar en la organización la movilidad del talento humano hacia el cumplimiento de objetivos y metas con altos estándares de excelencia.

Para lograr un ajuste entre los puestos y la estrategia institucional, es necesario que el análisis ocupacional parta de la definición del marco estratégico, con el fin de adaptar las tareas a los resultados que se esperan para cada uno de los procesos definidos en la estructura organizacional. La determinación de los puestos debe estar distribuida de manera que garantice el cumplimiento integral de los objetivos, como una forma de certificar el éxito colectivo. 
Fernández-López (2006) señala que "la gestión por competencias viene a introducir conceptos para flexibilizar la dirección de los empleados y poder adaptarla a las necesidades de la organización de la empresa” (p. 22).

La Organización Internacional del Trabajo (OIT) define las competencias como:

Capacidad de articular y movilizar condiciones intelectuales y emocionales en términos de conocimientos, habilidades, actitudes y prácticas, necesarias para el desempeño de una determinada función o actividad, de manera eficiente, eficaz y creativa, conforme a la naturaleza del trabajo. Capacidad productiva de un individuo que se define y mide en términos de desempeño real y demostrando en determinado contex to de trabajo y que no resulta solo de la instrucción, sino que, de la experiencia en situaciones concretas de ejercicio ocupacional. (citado por OTC, 2021, párr. 1)

De esta definición queda claro que la competencia no es una expectativa, sino es una capacidad demostrada, medible y cuantificable. Para la definición de las competencias que se ajustan a la institución, es necesario realizar lo siguiente:

- Tener claros los procesos de trabajo y sus resultados.

- Conocer los principios y valores que rigen a la organización.

- Definir las macro actividades por medio de las cuales se lograrán los resultados.

- Determinar los conocimientos que se requieren para llevar a cabo las actividades.

- Establecer cuáles habilidades y destrezas harán la diferencia en cada tarea.

- Identificar para cada actividad los principios y valores que rigen, alineados con los de la institución.

Una vez definido lo anterior, se propone la siguiente clasificación de las competencias:

- Transversales: son las competencias genéricas que deben estar presente en todos los puestos de la institución. Se relacionan de manera directa con la razón de ser de la organización. Por ejemplo, calidad, innovación y creatividad.

- Gerenciales: se refiere a las conductas, habilidades, destrezas que debe manifestar una persona para una dirección efectiva de equipos de trabajo. Entre estas están: liderazgo, empatía, comunicación, manejo de conflictos, ética, planeación, entre otras.

- Específicas: se relacionan con las habilidades, destrezas, actitudes y valores que se deben manifestar en el desempeño de un cargo.

Las habilidades blandas o habilidades de las personas se encuentran incluidas en los tres grupos de competencias mencionados, por cuanto son las que impulsan la inteligencia emocional. La participación activa en la definición de las competencias de las personas que realizan el trabajo, se convierte en factor clave para el éxito de este proceso, por cuanto es la forma más objetiva de obtener la información que relacione el perfil con las tareas de los puestos. La descripción del cargo en una gestión por procesos y competencias, debe contener, además de la definición de proceso y la actividad de manera específica, los resultados que se esperan. Para ello, la definición de indicadores contribuirá a generar un control efectivo sobre el cumplimiento de metas o, en su defecto, poder implementar ajustes de manera oportuna.

Captación del talento humano Los procesos de selección de personal estuvieron centralizados en los departamentos de recursos humanos por muchas décadas. Chiavenato (2020) los identifica como planteamientos tradicionales, en donde predomina el enfoque operacional y burocrático, con procesos estandarizados, llevados a cabo de manera secuencial. Además, indica que "el modelo es vegetativo por el hecho de que busca que se ocupe la vacante para mantener el statu quo de la organización” (p. 89), como característica de este modelo es que concentra toda la incorporación de personas en la gestión de talento humano.

El enfoque moderno, por el contrario, es más estratégico. El proceso de captar personas a la organización es responsabilidad del personal de línea, con la asesoría de los profesionales de la gestión de talento humano. Optar por este enfoque tiene la ventaja de que, al tener una participación más activa dentro del proceso de selección, los tomadores de decisiones tienen la oportunidad de formar sus propios equipos de trabajo, lo que podría generar mayor sinergia hacia la consecución de los objetivos y metas.

En este modelo estratégico de captación de talento no existen técnicas únicas aplicadas de manera uniforme para la determinación de la idoneidad en un puesto. Por el contrario, se produce un ajuste constante de acuerdo con las necesidades del proceso de trabajo de que se trate; es decir, una propuesta a la medida. 
Gestión del desempeño. El entorno en el que se desenvuelven las organizaciones en la actualidad tiene una característica común: la incertidumbre. Ante este panorama, es claro que sobreviven aquellas instituciones que logran obtener los resultados a pesar de las situaciones a las que se tienen que enfrentar en su cotidianidad. La única certeza que se puede afirmar es que es por medio de las personas que se logran los resultados, por lo que la incógnita a resolver sería: ¿cómo se puede medir la contribución que se hace a nivel individual para determinar el aporte en la consecución de los objetivos y metas?

Han sido diversas las propuestas planteadas para resolver esta interrogante. Algunas miden las características de las personas, otras relacionan estas características con las funciones del puesto. No obstante, lo que es una verdad en el contexto actual, es que es esencial conocer los resultados, por ello, un sistema de gestión del desempeño debe incluir, al menos, lo siguiente:

- Definición de metas por cumplir.

- Indicadores que permiten medir de manera objetiva esas metas.

- Competencias requeridas para la consecución de los objetivos.

- Tabla para medir resultados.

- Tabla para medir competencias.

- Establecimiento de escalas.

- Definición de cada criterio de la escala.

- Instrumentos de evaluación sencillos, acompañados de manuales accesibles, tanto para quien evalúa como para las personas evaluadas.

- Gestión de realimentación.

- Socialización.

Gestionar el desempeño requiere establecer procesos de planificación del trabajo, establecimiento de metas, incorporación de indicadores y la realimentación como proceso final para producir mejora continua. Visualizar de esta forma la evaluación del desempeño lleva a abandonar la antigua percepción punitiva, para transformarla en una oportunidad clara para corregir deficiencias y producir excelencia, o bien, trascender a otros niveles de la organización. Para que este proceso sea exitoso es requerimiento imprescindible que los procesos de sensibilización y comunicación sean abiertos, transparentes y objetivos.

Gestión de la capacitación o formación. Los procesos de capacitación o formación históricamente han estado envueltos en mitos que han ocasionado desidia en las personas que tienen a cargo la toma de decisiones a nivel organizacional. Un ejemplo de ello es pensar que la capacitación es un gasto o creer que solo es por medio de la capacitación que se corrigen los problemas del desempeño.

Por lo anterior, se propone lo siguiente para una institución de educación superior:

1) Un primer paso es determinar la forma en que se van a detectar las necesidades de capacitación o formación. Esta detección, en un modelo de gestión por competencias, debe estar orientada a conocer cuáles de las brechas existen entre el nivel de eficacia actual y el esperado para el desempeño individual (optimización). Asimismo, una necesidad de capacitación puede darse cuando se logre identificar que existen nuevos conocimientos que deben incorporarse, en virtud de un cambio en el proceso de trabajo. En una institución de educación superior, la gestión de talento humano debe ocuparse de proveer a la organización de personas críticas, actualizadas, con deseo de aprendizaje continuo y autónomos en su desempeño.

2) Planes de sucesión. El análisis de los índices de rotación y el estudio de los indicadores de empleo de la institución (característica de la población laboral) deben dar como resultado la necesidad de implementar programas de formación específicamente en puestos estratégicos, con el fin de identificar aquellos posibles talentos que puedan ocupar otras posiciones.

3) Nuevos procesos de trabajo o diversificación. Deben existir canales de comunicación continuos entre los tomadores de decisiones y la gestión de talento humano, con el fin de responder de manera oportuna a los cambios en los procesos, que requieran personal actualizado o con nuevas formaciones o competencias, que permitan agilizar la incorporación a nuevas ocupaciones. Esta forma estratégica de gestionar el talento permitirá reaccionar de manera adecuada a los cambios del entorno. 
Como puede observarse, en todos los casos la detección de las necesidades de capacitación o formación parten de una necesidad institucional. La administración de recursos humanos debe procurar mantener una batería de instrumentos actualizada, que le permita mantenerse en constante monitoreo de los cambios del entorno y, de ese modo, formular oportunamente la respuesta que la organización requiere. Los viejos programas de capacitación, prediseñados y sin visión de futuro, han dado paso a la investigación, al contacto con el ambiente y a la respuesta estratégica que se espera de su gestión.

Gestión de la compensación. En virtud del período de contracción de la economía por el que el mundo atraviesa, producto de las medidas de suspensión de actividades que los países han tenido que aplicar para contener la propagación del COVID-19, el tema de diseño de sistemas salariales ha quedado relegado en la discusión habitual, dando paso a la necesidad de obtener trabajo o estabilidad laboral. Sin embargo, todo diseño de sistema salarial debe contener al menos dos de las siguientes políticas: la equidad, referida al pago justo por los niveles de responsabilidad, complejidad, dificultad e impacto de las funciones que se realiza y la competitividad relativa a la ubicación del salario, con respecto a lo que se paga en puestos homólogos en instituciones del mismo ramo. La gestión de talento humano, en este aspecto, debe procurar mantener una estructura de puestos actualizada, de manera que provea instrumentos que garanticen la justicia en los niveles de compensación que se aplican en la organización.

En el modelo de gestión por competencias son diferentes aspectos los que deben determinarse con el fin de lograr una aplicación exitosa de la remuneración por competencias. Varela (2013) propone los siguientes:

4) 1. Un sistema que define aptitudes específicas, así como un proceso para determinar el pago de la persona con base en sus aptitudes y competencias.

5) 2. Un sistema de capacitación que permite a los empleados buscar y adquirir aptitudes.

6) 3. Un sistema formal de prueba de competencias.

7) 4. Un diseño de trabajo que permite a los empleados cambiar de puesto para promover la flexibilidad en la asignación de labores. (p. 277)

En todo caso, el éxito de la implementación de un buen sistema salarial depende de: a) análisis objetivo de la situación laboral y económica de la institución, b) amplia participación de los diferentes sectores en la discusión de las variables a incluir, c) la concientización de la factibilidad de la aplicación y d) políticas internas y externas sobre el salario en la organización.

\section{CONCLUSIÓN}

Con la evolución del concepto de "persona como centro de la organización”, la gestión de talento humano ha tenido que pasar de un rol pasivo, tramitador, tradicional y operativo, a ocupar un lugar estratégico, direccionando todos sus procesos hacia un liderazgo transformacional orientado a la innovación, la creatividad, la calidad y la incorporación de estándares de excelencia en el quehacer organizacional. Esta relevancia que ha adquirido en el contexto laboral actual requiere de un cambio paradigmático en lo que hasta ahora la administración del recurso humano había representado en las instituciones.

Un primer punto de discusión para lograr ese salto cualitativo en la gestión es el análisis crítico de lo que se hace, con el fin de medir de manera objetiva el aporte que se brinda a la organización, y se incorpora la revisión de criterios que se consideran diferenciadores de una efectiva gestión de talento humano. Es decir, el análisis de su cadena de valor, compuesta por: incorporación de la visión estratégica de la institución como norte de las actividades de talento humano, aporte y orientación de cada uno de los procesos a la consecución de la estrategia, niveles de coordinación y comunicación, flexibilidad, entre otros. Este análisis debe llevar a la toma de decisiones sobre los cambios urgentes que deben realizarse con el fin de ofrecer a las organizaciones el aporte estratégico que esta requiere. Huseild et al. (2005) resumen la función estratégica de los recursos humanos de la siguiente forma:

La visión de sociedad estratégica del departamento de recursos humanos debería centrarse primero en la responsabilidad compartida de la ejecución de la estrategia de la empresa, dado que allí es donde se puede dar la verdadera contribución estratégica de los recursos humanos. Esto significa que los profesionales de recursos humanos necesitan aceptar la responsabilidad de una serie de actividades que se escapan del alcance habitual de su gestión. (p. 87) 
Otro tema que debe ser analizado en este nuevo escenario es el protagonismo del accionar de la administración de recursos humanos. Desde esta perspectiva, es importante recordar que la gestión de talento tiene una función staff, es decir, su propósito fundamental es asesorar a las personas tomadoras de decisiones en los temas relacionados con el talento humano, y generar propuestas de ajuste o visión de futuro por medio del monitoreo constante de las variables que intervienen en el entorno en que se mueve la organización. Su eficacia debe ser medida justamente por los aportes que realiza a la estrategia, su capacidad para proponer de manera oportuna ajustes producto de potenciales problemas y la actualidad de sus procesos.

Esta nueva perspectiva de la administración de recursos humanos requiere también de un cambio en el perfil de quienes lideran estos procesos. En la administración tradicional bastaba con tener conocimientos específicos en el área para tener éxito. El entorno actual plantea una serie de habilidades, conductas, destrezas y aptitudes, las cuales deben estar manifiestas en el desempeño de la persona que tiene a cargo la función de dirigir esta actividad. A continuación, se plantea un resumen de las características consideradas como diferenciadoras, que deberán estar presentes en la persona que lidere los procesos de talento humano en una organización, principalmente en una institución de educación superior:

- Pensamiento sistémico. Zapata et al. (2008) indican que esta competencia se refiere a la toma de decisiones consensuada, se toman en consideración escenarios, opciones, consulta con personas expertas a nivel institucional en el tema, para una efectiva toma de decisiones.

- Credibilidad técnica. Definida por Alles (2009) como "la capacidad para generar confianza en los demás por su desempeño profesional y constituirse en un referente a quien consultar. Implica ser reconocido por poseer sólidos conocimientos y experiencia” (p. 175).

- Ética. Disposición para adaptar su actuación a los principios y valores establecidos en la institución. La persona debe ser muy rigurosa en mantener coherencia entre lo que dice y lo que hace.

- Facilidad en la comunicación. Implica la capacidad para saber interactuar con sus colaboradores y con el resto del personal de la organización.

- Habilidades sociales. Se refiere a diferentes competencias que deberá tener la persona que lidere el proceso de talento humano, relacionadas con el ámbito social, que se constituyen en estrategias de conducta que le permiten relacionarse con las demás personas de manera efectiva. Entre ellas están: empatía, asertividad y negociación.

Estas características, aunadas a un contexto institucional que permita el empoderamiento, el trabajo en equipo y el aprendizaje continuo, además de un clima laboral que facilite la comunicación y la información fluida y transparente, serán la clave para promover ambientes laborales que procuren la motivación organizacional mediante procesos de mejora y actualización. Los espacios laborales universitarios deben dinamizarse por medio del conocimiento. En este sentido, el aporte de la gestión de talento humano para promover entornos competitivos es invaluable, ya que establece acciones para que se logren transformar esos conocimientos en mejores prácticas y, de esa manera, coadyuvar en la articulación que debe existir entre la gestión interna y los cambios del entorno.

Por último, deben incorporarse a la gestión de talento humano indicadores que permitan medir el nivel aporte que realiza a los procesos clave institucionales. De forma que pueda cuantificarse la cadena de valor real de la gestión de talento humano y, en caso de encontrar desviaciones, se implementen acciones de mejora oportunamente. 


\section{REFERENCIAS}

Alles, M. (2009). Nuevo Enfoque Diccionario de Competencias. La trilogía. Editorial Gránica.

Ariza-Montes, J. A., Morales-Gutiérrez, A. C. y Morales-Fernández, E. (2004). Dirección y Administración Integrada de Personas. Fundamentos, procesos y técnicas en práctica. McGraw-Hill.

Bindé, J. (2005). Hacia las sociedades del Conocimiento. Informe Mundial de la UNESCO.

Bohlander, G. y Snell, S. (2008). Administración de Recursos Humanos. Cengage Learning Editores, S.A.

Chiavenato, I. (2011). Administración de Recursos Humanos. El Capital Humano de las Organizaciones (9.a ed.). McGraw-Hill.

Chiavenato, I. (2020). Gestión del Talento Humano. El nuevo papel de los recursos humanos en las organizaciones (5.a ed.). McGraw-Hill.

Dessler, G. y Varela, R. (2017). Administración de Recursos Humanos. Enfoque Latinoamericano (6.a ed.). Pearson Educación.

Dolan, S. L., Valle-Cabrera, R., Jackson, S. E. y Schuler, R. S. (2010). La Gestión de los Recursos Humanos. Cómo atraer, retener y desarrollar con éxito el capital humano en tiempos de transformación. McGraw-Hill.

Fernández-López, J. (2005). Gestión por competencias. Un modelo estratégico para la dirección de Recursos Humanos. Pearson Educación.

Fernández-López, J. (2006). Gestión por competencias. Un modelo estratégico para la dirección de recursos humanos. Pearson Educación.

Huseild, M. A., Becker, B. E. y Beatty, R. W. (2005). El cuadro de mando del capital humano. Gestión del Capital Humano. Editorial Deusto.

Ministerio de Planificación Nacional y Política Económica [MIDEPLAN]. (2020). Elementos básicos de la Cuarta Revolución Industrial y su impacto en Costa Rica. MIDEPLAN. https://documentos.mideplan.go.cr/share/s/ ctuNCOAWS2222DmU3M oOQ.

Murillo-Vargas, G., García-Solarte, M., y González-Campo, C. H. (2019). Propuesta de estructura organizacional para organizaciones intensivas de conocimiento: una caracterización desde los centros de excelencia. Revista Virtual Universidad Católica Del Norte, (58), 19-40. https://revistavirtual.ucn.edu.co/index.php/RevistaUCN/article/ view/1084.

Namdarian, L., Sajedinejad, A. y Bahanesteh, S. (2020). The Impact of Kowledge Management on Organizational Perfomance: A Structural Equation Modeling Study. Revista AD-minister, (37). http://web.a.ebscohost.com. cidreb.uned.ac.cr/ehost/pdfviewer/pdfviewer?vid=3\&sid=5df3cc3e-fcde-4017-aa94-d6fd 74833b15\%40sdc-vsessmgro2.

OTC. (2021). OTC Facilitadores Certificación Profesional. OTC. https://www.otece.com/otc-competencias-oit.html

Robbins, S. P. y Judge, T. A. (2017). Comportamiento Organizacional (17.a ed.). Pearson Prentice Hall.

Rodríguez-Ponce, E. (2009). El rol de las universidades en la sociedad del conocimiento y en la era de la Globalización: Evidencia desde Chile. Revista Interciencia, 34(11). http://ve.scielo.org/scielo.php?script=sci arttext\&pid=S0378-18442009001100013.

Tapasco-Alzate, O. A. y Giraldo-García, J. (2020). Asociación entre posturas administrativas de directivos y su disposición hacia la adopción del teletrabajo. Revista información Tecnológica, (31). https://scielo.conicyt.cl/scielo. php?pid=S0718-07642020000100149\&script $=$ sci_arttext.

Varela, R. (2013). Administración de la compensación. Sueldos, salarios y prestaciones. Pearson Educación.

Werther, W. B., Davis, K. y Guzmán, P. (2019). Administración del Capital Humano (8.a ed.). McGraw-Hill.

Werther, W. B. y Davis, K. (2005). Administración de Recursos Humanos. Administración de recursos humanos. El Capital Humano de las Empresas (6.a ed.). McGraw-Hill.

Zurbigriggen, C. y González-Lago, M. (2014). Innovación y co-creación: Nuevos desafíos para las políticas públicas. Revista de Gestión Pública, 3(2). https://revistas.uv.cl/index.php/rgp/article/view/2245/2197.

Zapata, L., Adrianenséns, M., Cárdenas, B., Frankle, L., Gómez, M. S. y Manrique, L. (2008). Aprendizaje Organizacional. McGraw-Hill. 